\title{
Analysis of Ground-Level Enhancements: Strong events are hard
}

Ilya Usoskin*

Space Climate Research Unit and Sodankylä Geophysical Observatory, University of Oulu, FIN-90014 Finland

E-mail: ilva.usoskindoulu.fi

Eleanna Asvestari

Space Climate Research Unit, University of Oulu, FIN-90014 Finland

Department of Physics, University of Helsinki, FIN-00014, Finland

\section{Teemu Willamo}

University of Helsinki, FIN-00014 Helsinki, Finland

\section{Agnieszka Gil}

Institute of Mathematics and Physics, Siedlce University, Stanislawa Konarskiego 2, 08-110 Siedlce, Poland

\section{Gennady A. Kovaltsov}

Ioffe Physical-Technical Institute, Politechnicheskaya st. 26, 194021 St. Petersburg, Russia

\section{Vladimir V. Mikhailov and Andrey Mayorov}

National research nuclear university (MEPhI), Kashirskoye sh. 31, 115409 Moscow, Russia

Ground Level Enhancements (GLEs) recorded by neutron monitor detectors are characterized by a variety of energy spectra of solar energetic particles (SEP), which vary between soft (as in August 1972) and hard (February 1956) ones. The aim of this work is to investigate the statistical relation between the hardness of the energy spectra and the event-integrated intensity. We calculated the event-integrated omnidirectional fluence of protons above $30 \mathrm{MeV}\left(F_{30}\right)$ and above 200 $\mathrm{MeV}\left(F_{200}\right)$ using energy spectra reconstructed from both ground-based and space-borne data. The ratio of the $F_{30}$-to- $F_{200}$ fluences is considered as an index of the hardness of the events spectra. The main results of this study is that all strong events (with the event-integrated intensity greater than $100 \% * \mathrm{hr}$ ) are characterized by a hard or very hard spectrum, while weak and moderate events do not show any clear pattern between the hardness and the intensity of the event.

35th International Cosmic Ray Conference - ICRC2017

10-20 July, 2017

Bexco, Busan, Korea

*Speaker. 


\section{Introduction}

Solar energetic particle (SEP) events, known also as solar particle storms, are sporadic events caused by solar flares or coronal mass ejections with great temporarily enhancements of the flux of energetic particles near Earth. Sometimes, the energy of SEPs can be sufficient to initiate an atmospheric cascade whose nucleonic component can be registered by ground-level neutron monitors (see, e.g., [四]). These events are conventionally called Ground Level Enhancements (GLE), which are listed since the mid-20th century (see the International GLE database http://gle.oulu.fi [[]]). Sometimes weaker events with a marginal response only at high-altitude polar neutron monitors (NMs) [3] , 团] can be recorded, however they are related to a subclass of GLE events, called sub-GLE, discussed elsewhere (Poluianov et al., this volume).

Since the exact energy spectrum of SEP events is hard to measure directly, it is often described by parametric models, e.g., an exponential over rigidity [1] or a power law with an exponential roll-off [ 6 ]. Probably, the most convenient and realistic shape is provided by the so-called Bandfunction [Q, [8], which is a double power law in rigidity with an exponential junction inbetween [Q]. Parameters of this spectrum have been defined for all major GLE events [《, [0].

The strength of SEP events is often quantified via the fluence of particles with energy above 30 $\mathrm{MeV}, F_{30}$, which is important for space weather applications [W]. However, this single index is not always representative because of the SEP spectra varying between hard and soft ones. In particular, ground based NMs are sensitive to higher energy part of the SEP spectrum and cannot assess the $F_{30}$ fluence. Although, the SEP spectrum was evaluated from satellite-borne data for the last decades (e.g., [四]), only ground-based data are available in the past, including cosmogenic radionuclides produced by cosmic rays in the atmosphere and stored in natural archives [12]. Such proxies are not sensitive to lower energy part of the spectrum but provide a measure for the $F_{200}$ fluence [[3]]. Here we present a statistical analysis of the hardness of energy spectra for GLE events.

\section{GLE strength}

The strength of a GLE is often characterized by the peak intensity, which however gives little clue about the integral intensity of the event, since the peaky impulsive events may be quite short in duration yielding lower intensity than gradual events with smaller peak flux but longer duration. Fig. $\square$ shows an example of GLE \#5 (23-Feb-1956) depicting the highest response (5116 \%) for Leeds NM and the greatest integral intensity $(I=5300 \% *$ hr $)$ for Ottawa NM. The integral intensity corresponds to the total fluence of SEPs rather than to the peak flux. For each GLE event we collected all available NM responses from the International GLE database (http://gle.oulu.fi) and averaged the integral intensity over all high-latitude (geomagnetic cutoff rigidity $<1.5 \mathrm{GV}$ ) and low altitude $(<200 \mathrm{~m}) \mathrm{NMs}$. The integral intensity $I$ varies from a few $\% * \mathrm{hr}$ to $5300 \% * \mathrm{hr}$. For each GLE we also defined the event-integrated fluences $F_{30}$ and $F_{200}$. As the measure of the softness of the SEP spectra we considered the ratio of $R=F_{30} / F_{200}$ calculated using the full spectra reconstructed in work [ [ $]$ ]. Higher values of $R$ imply a softer spectrum, viz. they have more soft particles of $\approx 30 \mathrm{MeV}$ compared to more energetic ones $(\approx 200 \mathrm{MeV})$ able to initiated a nucleonic cascade in the atmosphere. 


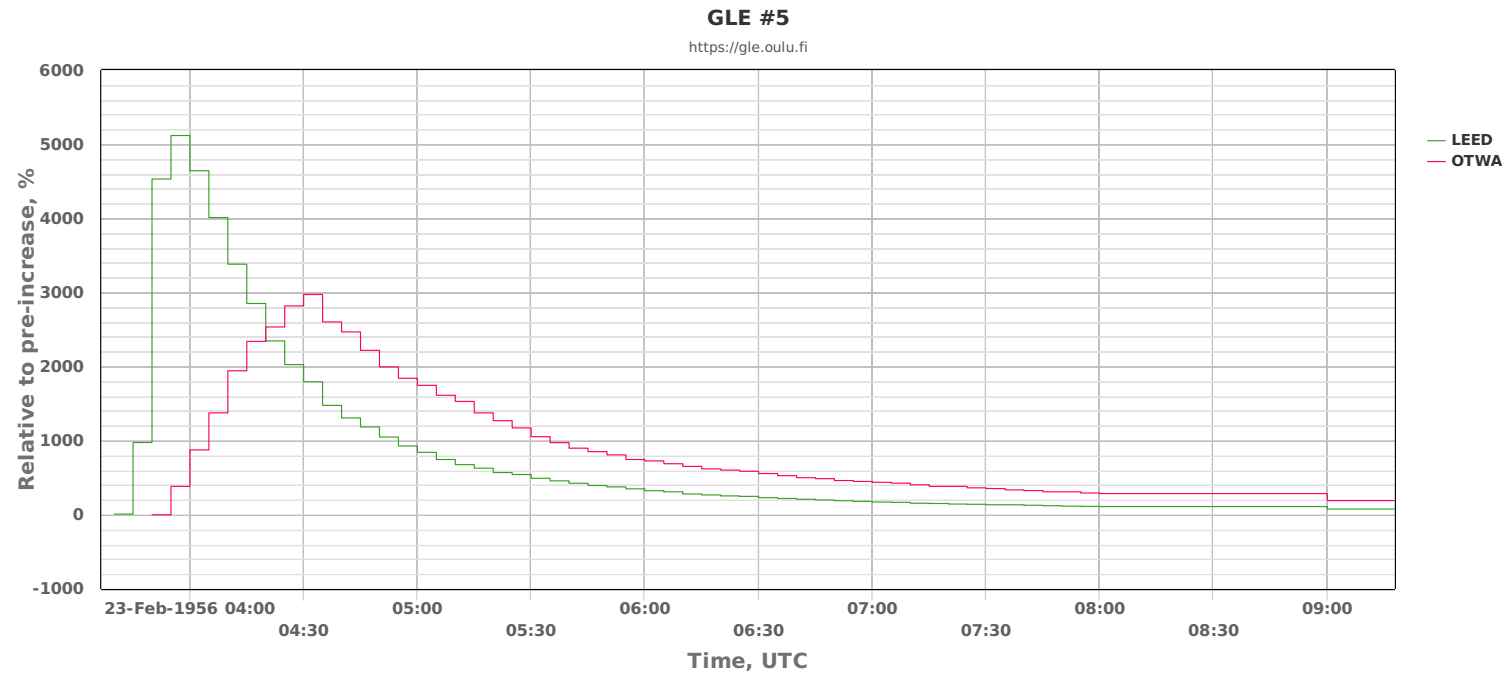

Figure 1: Percentage increase of the GLE \#5 (23-Feb-1956) measured by Leeds and Ottawa NMs. The plot was produced by http://gle.oulu.fi.

\section{Analysis}

We show in Figure $\square$ the $F_{30} / F_{200}$ ratio as a function of the GLE strength. We have arbitrary divided the events into three categories: weak, moderate and strong GLEs. Strong GLEs $(I>100$ $\% *$ hr) have hard energy spectra - the ratio is within the range 10-50. GLE \#5 has the ratio of about eleven, implying a very hard spectrum. Interestingly, the strongest known SEP event of 775 AD [144, ㄷ] had a very hard spectrum [16] as indicated by the red star in the Figure. For moderate GLE events $(10<I<100 \% * \mathrm{hr})$ the ratio takes a wide range of values, from 10 to 250 (the latter is for the very soft GLE \# 24 in August 1972), implying that there is no obvious relation for moderate events - they can have different spectra, from very hard to very soft. Interestingly, very weak GLEs $(I<10 \% *$ hr $)$ are harder than moderate events so that no soft events are found, but this may be caused by a selectional/observational bias.

The fact that strong GLE events tend to be hard may be related to a saturation of the lower energy particle flux, the so-called "streaming limit" [ㅍ, [18] caused by a possible resonance interaction between the particle flux and plasma waves at the interplanetary shock so that there is a limit for the flux of SEPs accelerated at one instance. An empirical estimate of the streaming limit is $F_{30}=(6-8) \cdot 10^{9} \mathrm{~cm}^{-2}$ [एव]. On the other hand, the $F_{200}$ fluence is not limited by this effect and keeps growing with the event's strength, thus the spectrum hardens for stronger events making the ratio $R$ to decrease.

\section{Conclusions}

Hardness of the SEP event-integrated energy spectra of GLE events for the last 60 years have been studied here. In addition, the strongest known historical SEP event of $775 \mathrm{AD}$ was also analyzed and found to be characterized by a very hard spectrum. It has been shown that all the strong GLE events with the event-integrated intensity $I>100 \% *$ hr have hard or very hard spectrum 


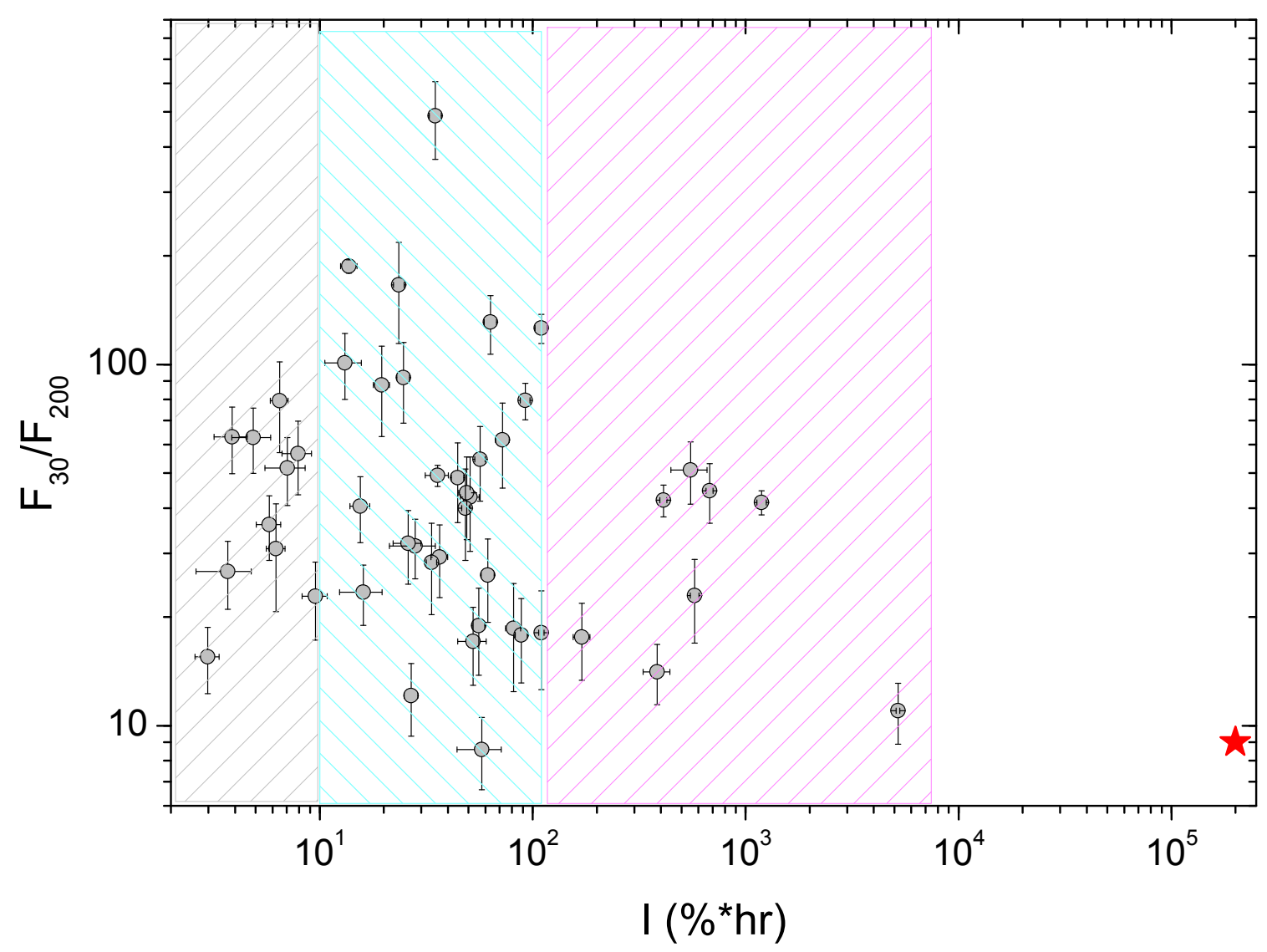

Figure 2: Ratio of $F_{30}$-to- $F_{200}$ fluence as a function of the GLE strength $I$ (grey dots). The red star represents the greatest historical SEP event of $775 \mathrm{AD}$. An arbitrary separation of the events to weak $(I<10)$, moderate $(10<I<100)$ and strong $(I>100 \% * \mathrm{hr})$ is indicated by color-hatched boxes.

spectrum with the ratio $R=F_{30} / F_{200}$ being in the range 10-50. Moderate events $(10<I<100$ $\% *$ hr) can have different spectra, from hard $(R<10)$ to very soft $(R>200)$ ones. Weak events also exhibit a tendency to be relatively hard. Although it is contrary to an intuitive expectation, it may be related to a selection/observation bias.

We speculate that the hardening of the spectrum of strong events may be achieved via the saturation (streaming limit) of the lower-energy particle acceleration/transport, while higher-energy particles are free of this limit. On the other hand, the existing observational and theoretical basis does not allow to make a conclusive statement on this.

\section{Acknowledgements}

This work was partly supported by the Center of Excellence ReSoLVE (Academy of Finland project No. 272157). A.G. acknowledges the Polish National Science Centre, decision number DEC-2016/22/E/HS5/00406. 


\section{References}

[1] M. A. Shea and D. F. Smart, Fifty Years of Cosmic Radiation Data, Space Sci. Rev. 93 (2000) 229-262.

[2] I. Usoskin, A. Ibragimov, M. A. Shea, and D. F. Smart, Database of Ground Level Enhancements (GLE) of High Energy Solar Proton Events, in 34th Intern. Cosmic Ray Conf. (ICRC2015), 2015.

[3] N. Thakur, N. Gopalswamy, H. Xie, P. Makelä, S. Yashiro, S. Akiyama, and J. M. Davila, Ground Level Enhancement in the 2014 January 6 Solar Energetic Particle Event, Astrophys. J. Lett. 790 (2014) L13.

[4] A. Belov, E. Eroshenko, O. Kryakunova, N. Nikolayevskiy, A. Malimbayev, I. Tsepakina, and V. Yanke, Possible ground level enhancements at the beginning of the maximum of Solar Cycle 24, J. Phys. Conf. Ser. 632 (Aug., 2015) 012063.

[5] P. S. Freier and W. R. Webber, Exponential Rigidity Spectrums for Solar-Flare Cosmic Rays, J. Geophys. Res. 68 (1963) 1605-1629.

[6] D. C. Ellison and R. Ramaty, Shock acceleration of electrons and ions in solar flares, Astrophys. J. 298 (1985) 400-408.

[7] D. Band, J. Matteson, L. Ford, B. Schaefer, D. Palmer, B. Teegarden, T. Cline, M. Briggs, W. Paciesas, G. Pendleton, G. Fishman, C. Kouveliotou, C. Meegan, R. Wilson, and P. Lestrade, BATSE observations of gamma-ray burst spectra. I - Spectral diversity, Astrophys. J. 413 (1993) 281-292.

[8] A. Tylka and W. Dietrich, A new and comprehensive analysis of proton spectra in ground-level encahnced (gle) solar particle events, in 31th International Cosmic Ray Conference, (Lodź, Poland), Universal Academy Press, 2009.

[9] I. G. Usoskin, G. A. Kovaltsov, I. A. Mironova, A. J. Tylka, and W. F. Dietrich, Ionization effect of solar particle GLE events in low and middle atmosphere, Atmos. Chem. Phys. 11 (2011) 1979-1988.

[10] A. J. Tylka and W. F. Dietrich, A Survey of Proton Spectra and Fluences above 1 GV in Ground-Level Enhanced (GLE) Solar Particle Events, EOS Trans. AGU 89(23), Jt. Assem. Suppl. 89(23) (2008) SH43C-03.

[11] R. Vainio, L. Desorgher, D. Heynderickx, M. Storini, E. Flückiger, R. B. Horne, G. A. Kovaltsov, K. Kudela, M. Laurenza, S. McKenna-Lawlor, H. Rothkaehl, and I. G. Usoskin, Dynamics of the Earth's particle radiation environment, Space Sci. Rev. 147 (2009) 187-231.

[12] J. Beer, K. McCracken, and R. von Steiger, Cosmogenic Radionuclides: Theory and Applications in the Terrestrial and Space Environments. Springer, Berlin, 2012.

[13] G. A. Kovaltsov, I. G. Usoskin, E. W. Cliver, W. F. Dietrich, and A. J. Tylka, Fluence Ordering of Solar Energetic Proton Events Using Cosmogenic Radionuclide Data, Solar Phys. 289 (2014) 4691-4700.

[14] F. Miyake, K. Nagaya, K. Masuda, and T. Nakamura, A signature of cosmic-ray increase in ad 774-775 from tree rings in Japan, Nature 486 (2012) 240-242.

[15] I. G. Usoskin, B. Kromer, F. Ludlow, J. Beer, M. Friedrich, G. A. Kovaltsov, S. K. Solanki, and L. Wacker, The AD775 cosmic event revisited: the Sun is to blame, Astron. Astrophys. 552 (2013) L3, [arxiv: [302,689]]. 
[16] F. Mekhaldi, R. Muscheler, F. Adolphi, A. Aldahan, J. Beer, J. McConnell, G. Possnert, M. Sigl, A. Svensson, H. Synal, K. Welten, and T. Woodruff, Multiradionuclide evidence for the solar origin of the cosmic-ray events of AD 774/5 and 993/4, Nature Comm. 6 (2015) 8611.

[17] D. V. Reames and C. K. Ng, Streaming-limited Intensities of Solar Energetic Particles on the Intensity Plateau, Astrophys. J. 723 (2010) 1286-1293.

[18] D. V. Reames, The Two Sources of Solar Energetic Particles, Space Sci. Rev. (June, 2013) 53-92, [arXiv: 1306,3608 ].

[19] K. McCracken, G. Dreschhoff, E. Zeller, D. Smart, and M. Shea, Solar cosmic ray events for the period 1561-1994: 1. identification in polar ice, 1561-1950, J. Geophys. Res. 106 (2001) 21585-21598. 